\title{
Der Alltag von Spitalärztinnen und -ärzten
}

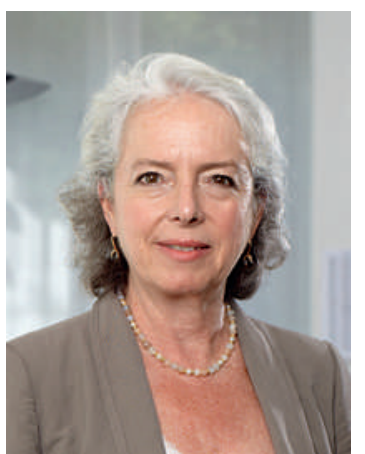

Seit dem 24. August läuft im Deutschschweizer Fernsehen eine DOK-Serie über Assistenzärzte, gezeigt wird der Alltag von sieben jungen Ärztinnen und Ärzten im Regionalspital Interlaken [1]. Die TV-Kritik zollt der Serie Beifall und lobt, dass die Dokumentation den TV-Zuschauern die Möglichkeit gebe, die Idealisierung der Halbgötter in Weiss zu überwinden und die Herausforderungen und Schwierigkeiten im Alltag von Ärzten in Weiterbildung besser zu verstehen.

Aus Sicht einer Berufskollegin ist diese DOK-Serie überaus gelungen und hilfreich, um das gegenseitige Verständnis zu verbessern!

Im derzeit angespannten politischen Klima fühlt sich der Bürger zwischen den Gesundheitsfachleuten und Krankenkassen hin- und hergerissen, und in der öffentlichen Diskussion geht es hauptsächlich um die Kosten. Manchmal kann beim Bürger sogar der Eindruck entstehen, dass die Ärzte Fehler vertuschen wollen und dass es ratsam sei, gegenüber dem Gesundheitspersonal eine defensive oder sogar streitbare Haltung einzunehmen. Diese DOK-Serie zeigt subtil und ohne übertriebenes Pathos, dass die Realität im Spital eine ganz andere ist.

\section{DOK-Serie über Assistenzärzte:}

\section{Ein solches Projekt ist nur zu begrüssen, da damit das gegenseitige Verständnis verbessert werden kann!}

In der Medizin besteht oftmals eine grosse Diskrepanz zwischen Technik und Menschlichkeit, die schwer zu erklären ist. In den letzten 30 Jahren war in der Medizin eine exponentielle Entwicklung zu verzeichnen. Die Zeiten, in denen der Hausarzt in paternalistischer Manier den Beipackzettel entfernte, bevor er seinem Patienten ein Medikament übergab, sind schon lange vorbei. Das Nichtwissen hatte auch manche Dinge offensichtlich vereinfacht! Die Tendenz, Gefahren zu ignorieren, ist zweifellos eine unserer Schwächen, und die unmittelbare Konfrontation mit einer Krankheit kann extreme Reaktionen hervorrufen: Zusammenbruch, Aggressionen oder Ablehnung der Behandlung.
Heutzutage sind die Behandlungen komplex und erfordern einen weitergehenden Einbezug («Patient Empowerment»). Die medizinischen Teams unternehmen entsprechende Anstrengungen, doch daraus resultieren auch Missverständnisse. Jeder vor uns hat seine persönliche Vorstellung von Gesundheit bzw. eine Theorie zu seiner Krankheit, die zwangsläufig subjektiv ist. Hinzu kommen der kulturelle Hintergrund, der unterschiedliche Bildungsstand und die Auswirkungen des Alters und des Geschlechts. Dadurch ist das gegenseitige Verständnis nicht immer einfach.

\section{Junge Ärztinnen und Ärzte agieren zwischen der klinischen Tätigkeit und der Forschung und stehen permanent unter dem Druck, der sich aus der verlangten Qualität und Wirtschaftlich- keit ergibt.}

Junge Spitalärzte sind somit mit verschiedenen Herausforderungen, mit den Anforderungen ihrer Weiterbildung sowie mit der Ungewissheit und den Ängsten angesichts von jederzeit möglichen Fehlern konfrontiert. Aufgrund ihrer anspruchsvollen Aufgaben stützen sie sich auf eine sehr strukturierte Hierarchie. Sie agieren zwischen der klinischen Tätigkeit und der Forschung und stehen permanent unter dem Druck, der sich aus der verlangten Qualität und Wirtschaftlichkeit ergibt. Ausserdem müssen sie in einer Gesellschaft, in der die Erziehung der Kinder zu einer schwierigen Herausforderung geworden ist, Arbeit und Familie unter einen Hut bringen.

Es ist zu begrüssen, dass diese DOK-Serie mehrere Episoden umfasst. Auch für die FMH ist es wichtig, dass sie in ihrem Zentralvorstand eine Vertreterin hat, welche die Anliegen der Spitalärztinnen und -ärzte noch besser einbringen kann.

Monique Gauthey, Fachärztin FMH, Mitglied des FMH-Zentralvorstands, Verantwortliche Ressort Spitalärzte

1 Serie «Die Assistenzärzte - Zeit der Bewährung», Freitagabend auf SF1, seit dem 24. August 